\title{
Structure and mechanical properties of a low-density AlCrFeTi medium entropy alloy produced by spark plasma sintering
}

\author{
D. Shaysultanov ${ }^{\text {a }}$, A. Nepapushev ${ }^{\mathrm{b}}, \mathrm{S}$. Zherebtsov $^{\mathrm{a}}$, D. Moskovskikh ${ }^{\mathrm{b}}$, N. Stepanov ${ }^{\mathrm{a}}{ }^{*}$ \\ ${ }^{a}$ Laboratory of Bulk Nanostructured Materials, Belgorod National Research University, Pobeda 85, 308015, Belgorod, Russia \\ ${ }^{\mathrm{b}}$ Center of Functional Nanoceramics, National University of Science and Technology, Leninskiy Prospekt 4, 119049, Moscow, Russia
}

\section{A R T I C L E I N F O}

\section{Keywords:}

Medium entropy alloys

Mechanical alloying

Spark plasma sintering

Microstructure

Mechanical properties

Phase stability

\begin{abstract}
A B S T R A C T
Bulk equiatomic AlCrFeTi medium entropy alloy was produced by mechanical alloying (MA) and spark plasma sintering (SPS). Powders with a homogeneous chemical composition composed of amorphous and bcc phases were obtained due to MA. Bulk, almost porosity free specimen of the alloy with the measured density of $5.53 \pm$ $0.07 \mathrm{~g} \times \mathrm{cm}^{-3}$ was obtained after SPS. A mixture of a (Fe, Ti)-rich C14 Laves phase, a Cr-rich bcc phase, and an Al-rich $\mathrm{L1}_{2}$ phase was found in the as-sintered specimens. The average size of grains/particles of the different phases was $115 \pm 100 \mathrm{~nm}$. Annealing at $1000^{\circ} \mathrm{C}$ resulted in some coarsening of the microstructure to the grain/ particle size of $140 \pm 120 \mathrm{~nm}$ and in an increase in the fraction of the bcc and $\mathrm{L} 1_{2}$ phases, yet the microstructure was found to be relatively stable. After SPS the AlCrFeTi alloy had a high microhardness of $1090 \pm 120 \mathrm{HV}$, which decreased after annealing to $870 \pm 70 \mathrm{HV}$. Compression tests have meanwhile revealed low ductility of the alloy after SPS - the alloy exhibited brittle fracture at room temperature and became ductile at only $800{ }^{\circ} \mathrm{C}$. After annealing the alloy demonstrated reasonable ductility already at $700{ }^{\circ} \mathrm{C}$. Also, the annealed alloy had a remarkable specific yield strength of $258 \mathrm{kPa} \times \mathrm{m}^{3} / \mathrm{kg}$ at $700{ }^{\circ} \mathrm{C}$. The experimental data on the phase composition of the alloy was compared with the Thermo-Calc predictions, and the relationships between the structure and properties of the alloy were discussed.
\end{abstract}

\section{Introduction}

The so-called high entropy alloys (HEAs) and related medium entropy alloys (MEAs) have attracted considerable attention from the materials scientists worldwide in the recent decade [1-4]. The nearly equiatomic compositions place the HEAs in the unexplored central positions of multicomponent phase diagrams $[4,5]$. As a result, the new alloying strategy allows creating alloys with unique microstructures and properties. It is believed that some HEAs can fill the "gaps" left by traditional alloys and find applications in demanding industries [6].

Although HEAs constitute a relatively new class of materials with a huge number of distinctively different compositions, the already introduced alloys can be classified into several categories [4]. The best-studied class of HEAs/MEAs is the alloys based on 3d transition metals (3D-TMHEAs/3D-TMMEAs) [4,7]. Typical alloys belonging to this family are AlCoCrCuFeNi [5] or CoCrFeMnNi [8,9]. The latter alloy is known for its exception fracture toughness at cryogenic conditions due to the development of nanotwinning in the single fcc solid solution structure [10]. The unique deformation behavior of CoCrFeMnNi and related alloys has been the subject of extensive investigations [7]. Generally, alloys based on 3d transition elements can have fcc phase, duplex fcc + bcc, or bcc-based structures, with the possible presence of different types of intermetallic phases [4,11-14]. In terms of chemical compositions, structures, and mechanical properties, they often can be considered as heavily alloyed "branches" of traditional steels or Ni-based superalloys.

Another well-developed alloy family is the so-called refractory HEAs/MEAs (RHEAs/RMEAs) [4,15]. RHEAs/RMEAs are based on refractory elements in a broad sense, i.e. $\mathrm{Cr}, \mathrm{Hf}, \mathrm{Mo}, \mathrm{Nb}, \mathrm{Ta}, \mathrm{Ti}, \mathrm{V}, \mathrm{W}$, and $\mathrm{Zr}$. Some non-refractory or non-metallic elements like $\mathrm{Al}$ or $\mathrm{Si}$ are often added. The initial purpose of RHEAs was the development of new materials for high-temperature applications in the aerospace sector [16]. Indeed, some of the alloys have demonstrated remarkable strength at high temperatures, outperforming even Ni-based superalloys in terms of temperature capacity [17-20]. However, identification of RHEAs/RMEAs with a balanced combination of high-temperature strength, ductility and toughness, and environmental resistance, remains a significant problem. RHEAs tend to have bcc-based structures, with the

\footnotetext{
* Corresponding author.

E-mail addresses: stepanov@bsu.edu.ru, stepanov.nikita@icloud.com (N. Stepanov).
} 
possible presence of $\mathrm{B} 2$, Laves, $\mathrm{M}_{\mathrm{x}} \mathrm{Al}$, and other phases [15]].

The so-called low-density HEAs/MEAs (LDHEAs/LDMEAs) represent third, yet much less studied alloy family [4,21]. There is no acknowledged definition of LDHEAs/LDMEAs in terms of chemical composition yet, however, it is believed that their density must be below $7 \mathrm{~g} / \mathrm{cm}^{3}[22$, 23]. Elements with a low density such as $\mathrm{Al}, \mathrm{Be}, \mathrm{Li}, \mathrm{Mg}$, Ti, and $\mathrm{Zn}$ are usually used, as well, however, as other elements with higher density. Therefore, some alloys based on 3d elements [24] and RHEAs [25,26] can be also considered as LDHEAs.

Due to a high diversity of the chemical compositions, it is difficult to establish regularities in the phase structure of LDHEAs/LDMEAs. However, as most of the elements with the low density have very different atomic and crystal structures, they tend to form intermetallic compounds due to limited mutual solubility [27]. As a result, many alloys have complex multiphase structures and limited ductility [28,29]. Moreover, the boiling and melting temperatures of the low-density elements also vary strongly. This makes the production of LDHEAs/LDMEAs using standard metallurgical practices rather complicated [4].

Powder metallurgy can be used to produce complex alloys successfully; among different powder metallurgy methods, a combination of mechanical alloying (MA) with spark plasma sintering (SPS) is a promising route to obtain different HEAs/MEAs [30-35]. The prepared alloys had almost $100 \%$ density, fine (often nano-grained) and relatively stable microstructure, and promising mechanical and functional properties. However, the production of LDHEAs/LDMEAs by MA followed by SPS has not been comprehensively studied so far [36]. Thus, the objectives of the present study are to produce by powder metallurgy, i.e. by MA with subsequent SPS, the low-density ( $\rho \approx 5.23 \mathrm{~g} / \mathrm{cm}^{3}$, estimated by a rule of mixtures) AlCrFeTi medium entropy alloy and (ii) to examine microstructure and mechanical properties of the alloy. Note that mechanical alloying of the AlCrFeTi mixture was reported in Ref. [37], but to the best of authors' knowledge, the structure and properties of a bulk alloy were never reported. Considering the non-equilibrium nature of the MA and SPS processes, specific attention is given to the estimation of the obtained microstructure and properties stability.

\section{Materials and methods}

The commercial powders of aluminum ( $99.7 \mathrm{wt} \%$, particle size $<30$ $\mu \mathrm{m})$, chromium ( $99.7 \mathrm{wt} \%$, particle size in the range $10-30 \mu \mathrm{m}$ ), iron (99.9 wt\%, particle size $<75 \mu \mathrm{m}$ ) and titanium (99.5 wt\%, particle size $<45 \mu \mathrm{m}$ ) were used as the precursors. An $18 \mathrm{~g}$ batch of an initial stoichiometric Al:Cr:Fe:Ti (1:1:1:1 mol) mixture was used for mechanical alloying at $694 \mathrm{rpm}$ (jar rotated at $1388 \mathrm{rpm}$ ) for $60 \mathrm{~min}$ in a watercooling double-station planetary ball mill (Activator 2S, Activator, Russia), which is high energy planetary ball mill and allows reaching centrifugal acceleration more than $90 \mathrm{~g}$ [38]. The milling ball (stainless steel, $6 \mathrm{~mm}$ diameter) to powder mixture weight ratio was 20:1. Before the milling, a stainless steel jar of $250 \mathrm{ml}$ capacity was vacuumed, filled by $99.98 \%$ pure argon with a pressure of $4 \mathrm{~atm}$ and then sealed. It is critical to note that the powders became pyrophoric, that is fresh as-milled powders could self-ignite after opening the jars. The powders were unloaded from jars and loaded to graphite die for SPS in a glove box in an argon atmosphere.

Cylindrical graphite die (the inner diameter of $30 \mathrm{~mm}$ ) was filled with a desired amount of the mixed powder. Then the powder was consolidated using a SPS apparatus (Labox 650, Sinter Land, Japan) using the following parameters: heated in vacuum (15-20 Pa) at the rate of $100{ }^{\circ} \mathrm{C} / \mathrm{min}$, the temperature of $900{ }^{\circ} \mathrm{C}$, under uniaxial loading of 50 $\mathrm{MPa}$, and dwell time of $10 \mathrm{~min}$. After sintering, the specimen was free cooled in vacuum; cooling to room temperature took $\sim 15 \mathrm{~min}$. Discshaped samples of $30 \mathrm{~mm}$ in diameter and $7 \mathrm{~mm}$ thick were cut and polished for further tests.

The actual chemical composition of the alloy (measured using energy dispersive spectrometry (EDS); the scan area was $\sim 1 \times 1 \mathrm{~mm}^{2}$ ) is presented in Table 1 . The measured composition closely corresponded to the nominal one. The density of the alloy was determined using hydrostatic weighting (Archimedes' principle) of three samples measured $4 \times 4 \times 6 \mathrm{~mm}^{3}$. The measured density was $5.53 \pm 0.07 \mathrm{~g} \times \mathrm{cm}^{-3}$. Some specimens after SPS were annealed at $1000{ }^{\circ} \mathrm{C}$ for $24 \mathrm{~h}$ with further air cooling. Before the annealing, the samples were sealed in a vacuumed $\left(10^{-2}\right.$ torr) quartz tube filled with titanium chips to prevent oxidation.

Structure of the alloy in different conditions was studied using X-ray diffraction (XRD), transmission (TEM) and scanning (SEM) electron microscopy; the microscopes (both TEM and SEM) were equipped with EDS units. XRD analysis was performed using a Rigaku diffractometer with $\mathrm{CuK} \alpha$ radiation. Specimens for SEM observations were prepared by careful mechanical polishing. SEM back-scattered electron (BSE) images were obtained using a FEI Quanta 600 FEG microscope.

Specimens for TEM analysis were prepared by conventional twin-jet electro-polishing of mechanically pre-thinned to $100 \mu \mathrm{m}$ foils, in a mixture of methanol (600 ml), butanol $(360 \mathrm{ml})$, and perchloric acid (60 $\mathrm{ml}$ ) at a temperature of $-35{ }^{\circ} \mathrm{C}$ and an applied voltage of $29.5 \mathrm{~V}$. TEM investigations were performed using a JEOL JEM-2100 microscope with an accelerating voltage of $200 \mathrm{kV}$.

Vickers microhardness tests were conducted at room temperature using $300 \mathrm{~g}$ load. At least 10 measurements per each data point were made. Nanoindentation was performed using a Shimadzu DUH-211s Dynamic Ultra Micro Hardness Tester with a Berkovich tip. At least ten indents were performed on each phase avoiding any influence from other phases. The maximum load of $50 \mathrm{mN}$ was applied for $5 \mathrm{~s}$; a loading rate was $6.662 \mathrm{mN} / \mathrm{s}$. The hardness was calculated using the Oliver and Pharr method [39].

Isothermal compressions of rectangular specimens measured $4 \times 4$ $\times 6 \mathrm{~mm}^{3}$ were carried out at room temperature and 600,700 , or $800{ }^{\circ} \mathrm{C}$ using an Instron 300LX test machine equipped with a radial furnace. The specimens were placed into the preheated to the testing temperature furnace and held for $\sim 10 \mathrm{~min}$ to equilibrate the temperature before testing. The temperature was controlled by a thermocouple attached to a side surface of the specimen. The initial strain rate was $10^{-4} \mathrm{~s}^{-1}$.

The equilibrium phase diagram was constructed using a Thermo-Calc 2020a software and a TCHEA3 database.

\section{Results}

\subsection{Powder after mechanical alloying}

Fig. 1a shows the XRD pattern of the AlCrFeTi alloy powder after MA. Several wide peaks are visible; all the observed peaks can be ascribed to a bcc solid solution with the lattice parameter of $0.291 \mathrm{~nm}$. Besides, a typical of amorphous structures "halo" was observed at low $2 \theta$ angles. SEM investigation has revealed that the obtained powder with a size of about 4-7 $\mu \mathrm{m}$ had a polygonal shape with sharp edges. The EDS maps (Fig. 1c-f) suggested a homogeneous distribution of alloying elements inside the powder. Finally, TEM examination of the obtained powder has also revealed the presence of amorphous matrix (amorphous "halo" at the selected area electron diffraction (SAED) pattern) with some nanoparticles/grains of the bcc phase, also evident from the diffraction maximums identified in the SAED pattern (Fig. 1g). EDS scanning has not revealed noticeable variations between the chemical compositions of the amorphous and bcc phases.

Table 1

Chemical composition of the typical structural constituents in the AlCrFeTi alloy after SPS (numbered in Fig. 2c), as per SEM-EDS measurement.

\begin{tabular}{|c|c|c|c|c|c|}
\hline \multicolumn{2}{|c|}{ Structural constituent } & \multicolumn{4}{|c|}{ Elements, at.\% } \\
\hline$\#$ & Designation & $\mathrm{Al}$ & $\mathrm{Ti}$ & $\mathrm{Fe}$ & $\mathrm{Cr}$ \\
\hline 1 & Matrix & 26.4 & 25.4 & 26.1 & 22.1 \\
\hline 2 & Light particles & 25.1 & 16.9 & 21.4 & 36.5 \\
\hline 3 & Dark particles & 33.3 & 23.4 & 21.8 & 21.5 \\
\hline \multicolumn{2}{|c|}{ Actual composition } & 26.7 & 23.2 & 25.9 & 24.2 \\
\hline
\end{tabular}




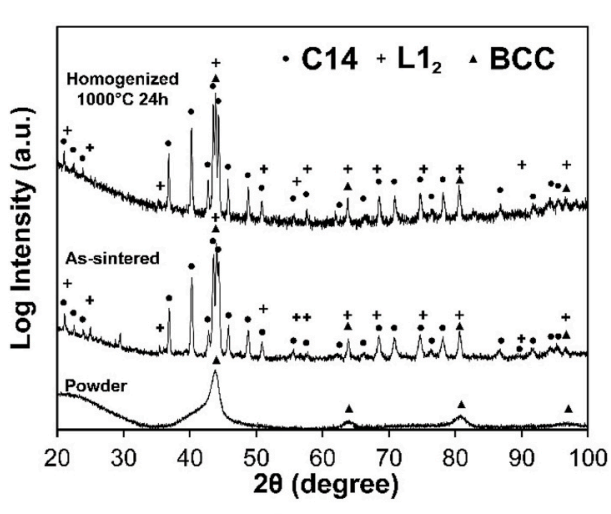

a

$\mathrm{Ti} K \alpha 1$

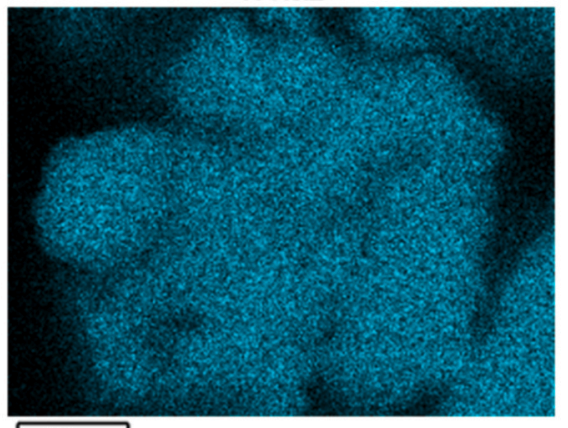

$2.5 \mu \mathrm{m}$

$\mathrm{Cr} \mathrm{K} \alpha 1$

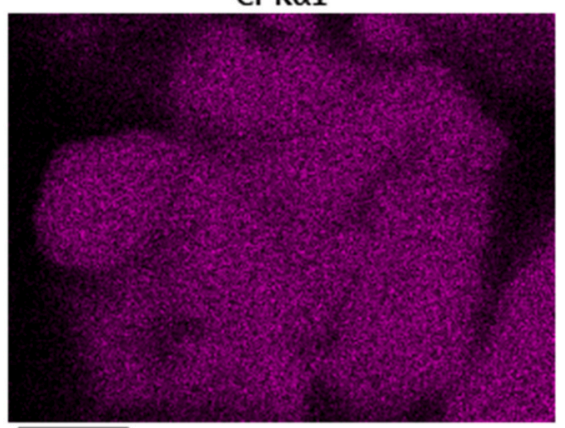

$\longdiv { 2 . 5 \mu \mathrm { m } }$

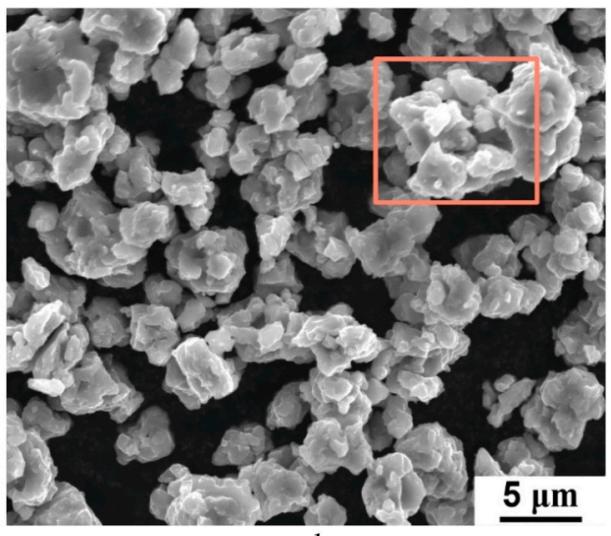

b

Al Ka1

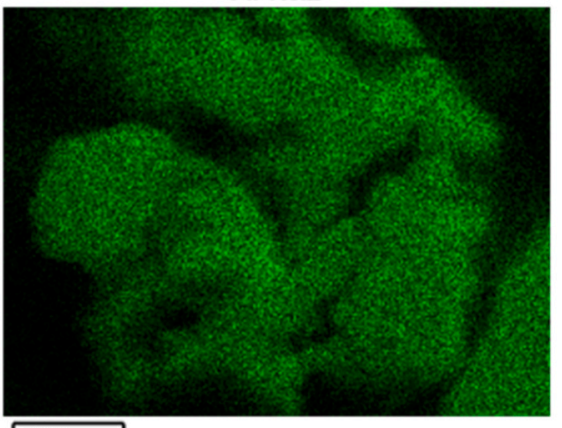

$\longdiv { 2 . 5 \mu \mathrm { m } }$

d

Fe K $\alpha 1$

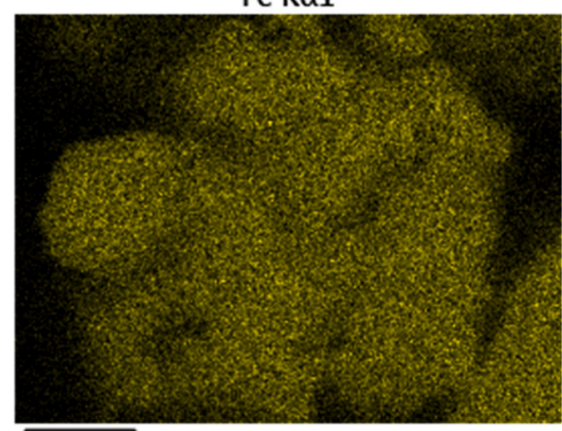

$\longdiv { 2 . 5 \mu \mathrm { m } }$

e

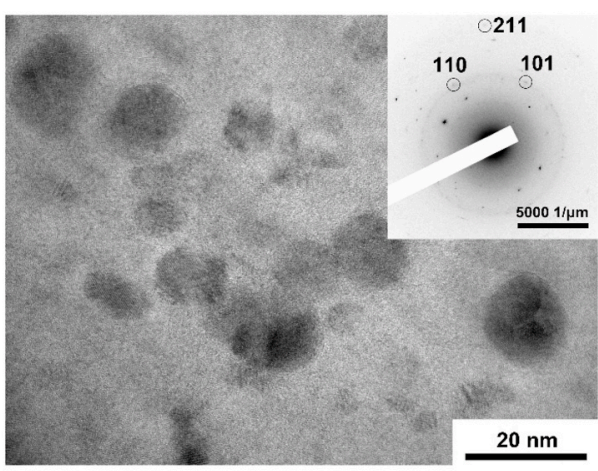

g 


\subsection{Structure after spark plasma sintering}

As per XRD results (Fig. 1a) SPS changed the phase composition of the AlCrFeTi alloy significantly. In addition to the previously existed bcc phase, multiple peaks attributed to either the C14 (hexagonal) Laves or (and) $\mathrm{L} 1_{2}$ phases were detected. The lattice parameters of the bcc phase increased slightly to $0.292 \mathrm{~nm}$. The lattice parameters of the Laves phases were $\mathrm{a}=0.490 \mathrm{~nm}$ and $\mathrm{c}=0.795 \mathrm{~nm}$, while the $\mathrm{L} 1_{2}$ phase had a lattice parameter of $0.714 \mathrm{~nm}$.

SEM observations have revealed quite a complex microstructure in the alloy after SPS (Fig. 2). In a low magnification image (Fig. 2a), darker and lighter areas were found. The lighter areas mostly appeared as individual "particles" separated by the darker areas. Both these areas were close to the equiatomic composition, yet somewhat (by 1 at.\%) higher Al concentration was found in the darker areas, while the percentage of $\mathrm{Fe}$ was similarly higher in the lighter areas. Note that very limited porosity $(<1 \%$ ) was found by metallographic observations; some pores are identified with arrows in Fig. 2a.

A higher magnification image shows that the darker and lighter areas visible in Fig. 2a differ from each other by their phase compositions (Fig. 2b and c). Three phases with different contrasts were revealed using BSE: a grey matrix phase (indicated as \#1 in Fig. 2c), light particles (\#2), and dark particles (\#3). More dark particles were found in the darker areas on low magnification areas (Fig. 2b) and vice versa.

Note that the particles containing lighter elements appear darker in the BSE images. The appearance of different phases is consistent with their chemical composition as determined by EDS analysis (Table 1): the grey matrix had a close to equiatomic composition (slightly enriched with $\mathrm{Fe}$ and $\mathrm{Ti}$ ), the dark particles were enriched with $\mathrm{Al}$, and the light particles - with $\mathrm{Cr}$. The estimated volume fraction of the light and dark particles was $10 \%$ and $7 \%$, respectively. The size of the light and dark particles was, respectively, $0.21 \pm 0.09 \mu \mathrm{m}$ and $0.28 \pm 0.08 \mu \mathrm{m}$.

TEM analysis was performed further to characterize the microstructure of the alloy in more detail (Fig. 3). The bright-field image has revealed a complex mixture of grains/particles of different phases. The average grain/particle size was $115 \pm 100 \mathrm{~nm}$. Boundaries of the grains/ particles were rather diffuse; a complex contrast inside some of the grains/particles can imply internal stresses. The correlative analysis using EDS measurements of chemical composition and determination of the crystal structure via SAED patterns allows accurate identification of the constitutive phases. The phases were: (i) C14 Laves phase matrix enriched with $\mathrm{Ti}$ and $\mathrm{Fe}$; (ii) bcc particles, enriched with $\mathrm{Cr}$ (visible as the dark particles in the SEM-BSE images (Fig. 2); (iii) $\mathrm{L1}_{2}$ particles, enriched with $\mathrm{Al}$ (visible as the light particles). No signs of the amorphous phase were found.

Some discrepancies between the results of the chemical analysis presented in Tables 1 and 2 can be attributed to a limited spatial resolution of the SEM-EDS system, not sufficient for accurate measurements of the chemical composition of fine grains/particles of the different phases. Nevertheless, SEM data is used hereafter for the determination of grain/particle sizes and fractions since it provides better statistics and allows easier distinguishing between the different phases due to the material contrast.

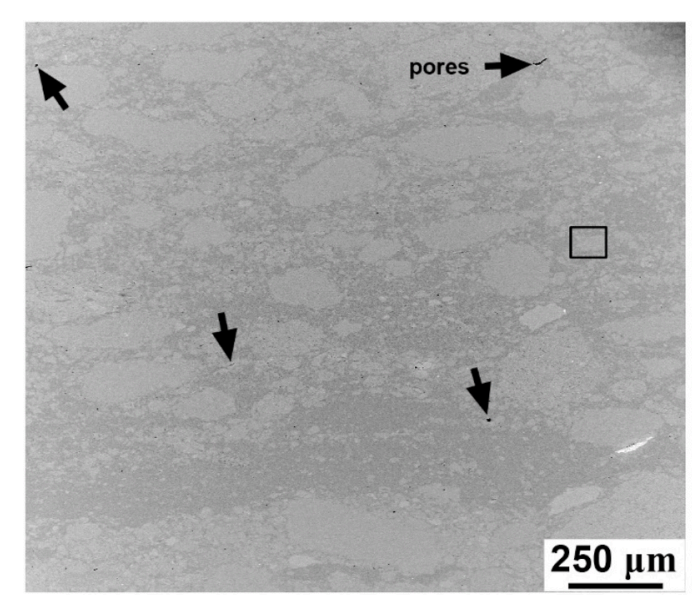

a

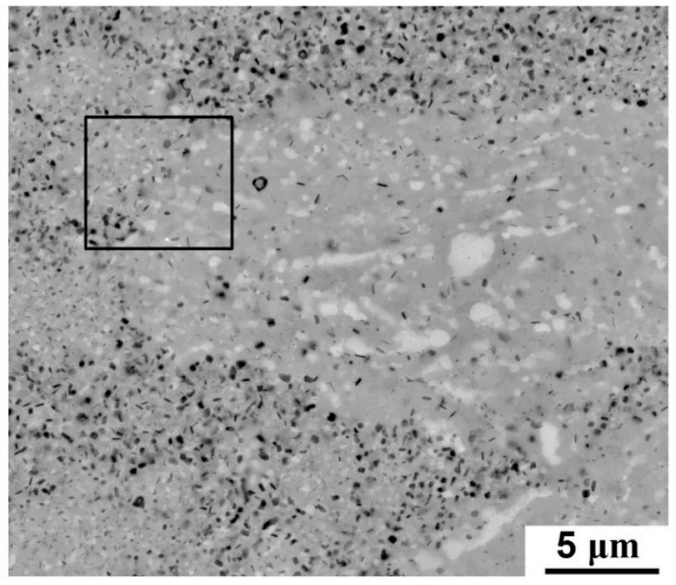

b

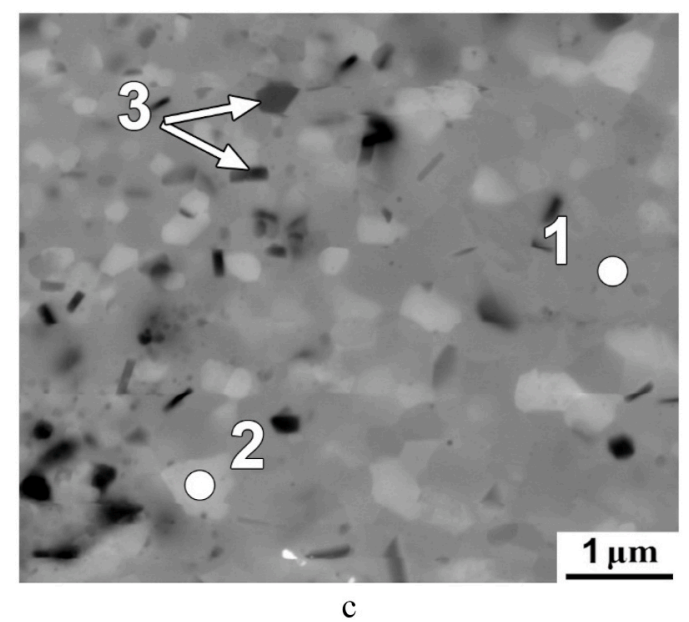

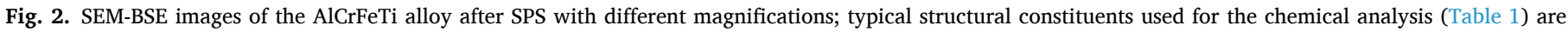
indicated in Fig. 2c. The observation areas for a higher magnification images are indicated with black boxes. Pores were indicated with black arrows. 


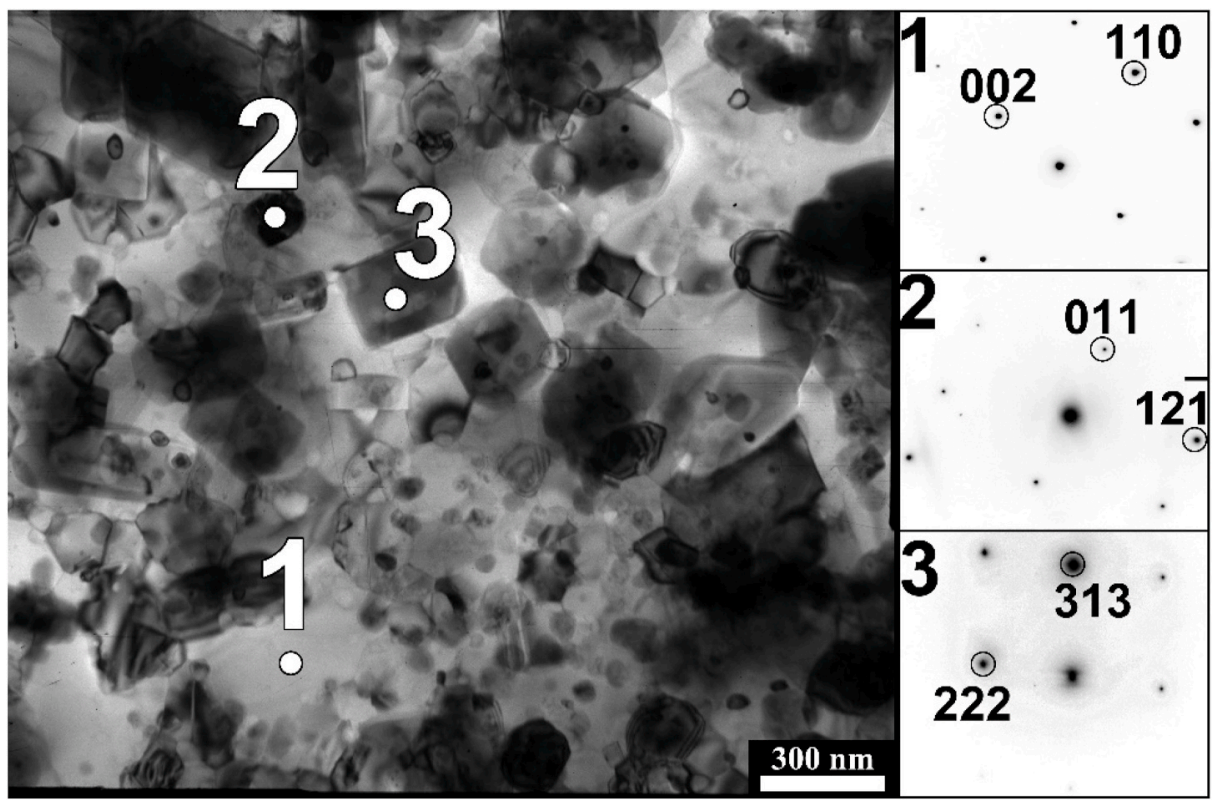

Fig. 3. TEM bright-field image of the microstructure of the AlCrFeTi alloy after SPS and corresponding selected area electron diffraction (SAED) patterns taken from representative structural constituents, denoted with numbers.

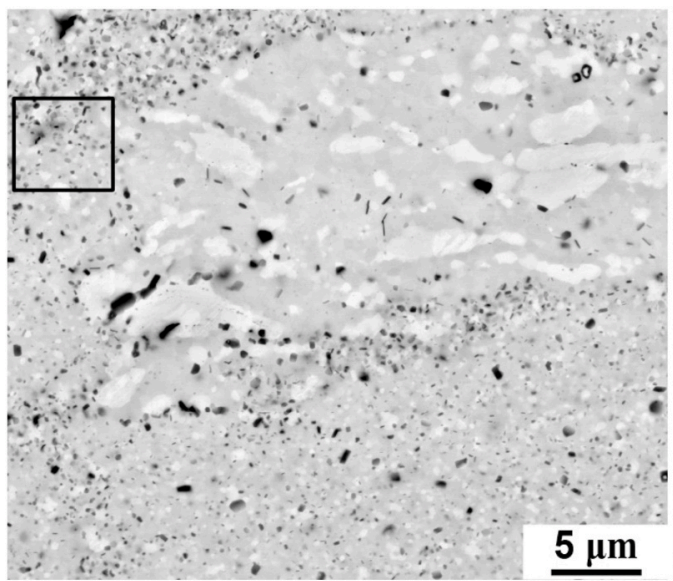

a

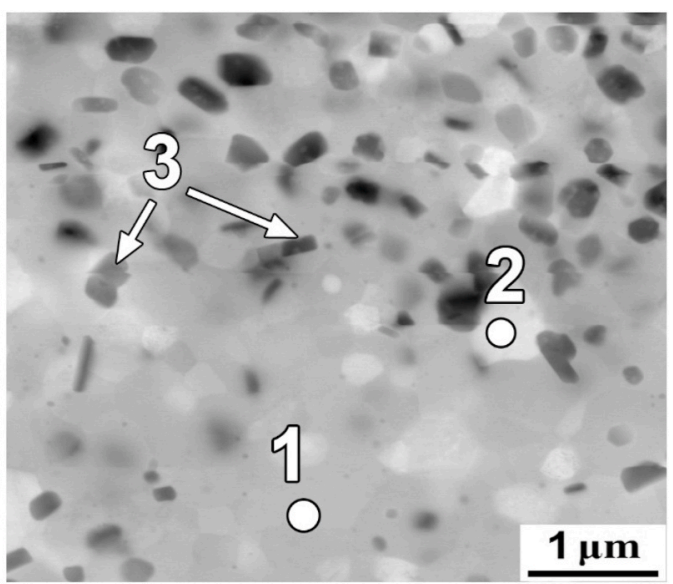

b

Fig. 4. SEM-BSE images with different magnifications of the AlCrFeTi alloy after SPS and annealing at $1000^{\circ} \mathrm{C}$ for $24 \mathrm{~h}$; typical structural constituents used for chemical analysis (Table 3 ) are denoted in Fig. $4 \mathrm{~b}$. The observation area for a higher magnification image is indicated with a black box.

Table 2

Chemical composition of the typical structural constituents in the AlCrFeTi alloy after SPS (numbered in Fig. 3), measured using TEM-EDS.

\begin{tabular}{llllll}
\hline \multicolumn{2}{l}{ Structural constituent } & \multicolumn{2}{l}{ Elements, at.\% } & & \\
$\#$ & Crystal structure & $\mathrm{Al}$ & $\mathrm{Ti}$ & $\mathrm{Fe}$ & $\mathrm{Cr}$ \\
1 & C14 Laves & 13.9 & 32.8 & 31.5 & 21.8 \\
2 & $\mathrm{bcc}$ & 16.5 & 13.6 & 19.5 & 50.4 \\
3 & $\mathrm{L1}_{2}$ & 59.9 & 17.1 & 13.0 & 10.0 \\
\hline
\end{tabular}

\subsection{Microstructure stability}

To estimate the stability of the microstructure produced by SPS, the alloy was annealed at $1000^{\circ} \mathrm{C}$ for $24 \mathrm{~h}$. However, the annealing had quite a limited effect on the structure of the AlCrFeTi alloy. XRD has not detected any noticeable changes in comparison with the as-sintered condition (Fig. 4). SEM investigations have also not revealed significant changes in the microstructure. Three typical constituents were found: the grey Laves phase matrix (\#1 in Fig. 4b), the light bcc particles
(\#2), and the dark $\mathrm{L1}_{2}$ particles (\#3). The fractions of the phases were somewhat affected by annealing treatment: the amount of the bcc phase and $\mathrm{L}_{2}$ phases increased to $13 \%$ and $11 \%$, respectively. Some insignificant coarsening was also observed: the size of the bcc particles and $\mathrm{L} 1_{2}$ phase particles increased to $0.24 \pm 0.13 \mu \mathrm{m}$ and $0.30 \pm 0.10 \mu \mathrm{m}$, respectively. The chemical composition of the phases was very close to that after SPS (compare Tables 1 and 3).

Table 3

Chemical composition of the typical structural constituents in the AlCrFeTi alloy after SPS and annealing at $1000^{\circ} \mathrm{C}$ for $24 \mathrm{~h}$, denoted with numbers in Fig. $4 \mathrm{~b}$, as per SEM-EDS measurement.

\begin{tabular}{|c|c|c|c|c|c|}
\hline \multicolumn{2}{|c|}{ Structural constituent } & \multicolumn{4}{|c|}{ Elements, at.\% } \\
\hline \# & Designation & $\mathrm{Al}$ & $\mathrm{Ti}$ & $\mathrm{Fe}$ & $\mathrm{Cr}$ \\
\hline 1 & Matrix (C14 Laves) & 21.2 & 26.2 & 34.0 & 18.6 \\
\hline 2 & Light particles (bcc) & 20.4 & 10.7 & 22.2 & 46.7 \\
\hline 3 & Dark particles $\left(\mathrm{L}_{2}\right)$ & 32.3 & 23.1 & 23.1 & 21.5 \\
\hline \multicolumn{2}{|c|}{ Actual composition } & 26.7 & 23.2 & 25.9 & 24.2 \\
\hline
\end{tabular}


TEM investigations (Fig. 5) have also not revealed significant changes in the microstructure. Some coarsening of grains/particles can be noted; their size increased to $140 \pm 120 \mathrm{~nm}$. Sharp, clean grain/ interphase boundaries also must be noted. The same 3 phases, namely (Fe, Ti)-rich C14 Laves phase matrix, Cr-rich bcc particles, and Al-rich $\mathrm{L}_{2}$ particles were found (Fig. 5, Table 4).

\subsection{Mechanical properties}

Measurements have revealed a high microhardness of the alloy after SPS of $1090 \pm 120 \mathrm{HV}$ (Fig. 6a), which decreased after annealing at $1000^{\circ} \mathrm{C}$ for $24 \mathrm{~h}$ to $870 \pm 70 \mathrm{HV}$ (Fig. $6 \mathrm{~b}$ ). For a more in-depth analysis, nanoindentation was performed to examine the mechanical response of individual phases. The following observations were made: (i) the C14 Laves phase is the hardest and the stiffest, the L12 phase has similar to the Laves phase Young's modulus but 20-40\% lower nanohardness, and the bcc phase has two times lower hardness than the L12 phase and $\sim 50 \%$ lower Young's modulus than the two other phases; (ii) annealing has resulted in $\sim 20 \%$ reduction in the hardness of the Laves phase, while the hardness of the other phases was hardly affected; (iii) annealing has barely affected Young's moduli of the constitutive phases.

In addition, compression tests at different temperatures were conducted (Fig. 7). At room temperature, the alloy after SPS fractured at $\sim 1100 \mathrm{MPa}$ immediately after yielding (Fig. 7a). Similar behavior was observed at $600{ }^{\circ} \mathrm{C}$, yet the alloy fractured at even lower stresses of $\sim 550 \mathrm{MPa}$. Some signs of ductility were revealed during testing at 700 ${ }^{\circ} \mathrm{C}$; the alloy had the yield strength of $880 \mathrm{MPa}$ but fractured soon after yielding. Finally, the alloy became ductile at $800{ }^{\circ} \mathrm{C}$ and had not fractured even after $50 \%$ height reduction. Yet, the yield strength of the alloy was low - $245 \mathrm{MPa}$. After yielding, the flow stress slowly increased with strain.

Annealing did not affect noticeably the mechanical behavior of the $\mathrm{AlCrFeTi}$ alloy both at room temperature and $600^{\circ} \mathrm{C}$ (Fig. $7 \mathrm{~b}$ ): the alloy fractured in the elastic region at $\sim 1100 \mathrm{MPa}$ and $\sim 350 \mathrm{MPa}$, respectively. Meanwhile, the alloy demonstrated a combination of reasonable ductility of $9.5 \%$ and a high yield strength of $1430 \mathrm{MPa}$. After yielding, the alloy showed a short work hardening stage and reached the peak stress of $1690 \mathrm{MPa}$. A flow softening stage and fracture was observed afterward. At $800{ }^{\circ} \mathrm{C}$, the annealed alloy behaved similarly to that after SPS; the yield strength was somewhat higher (270 MPa), however.

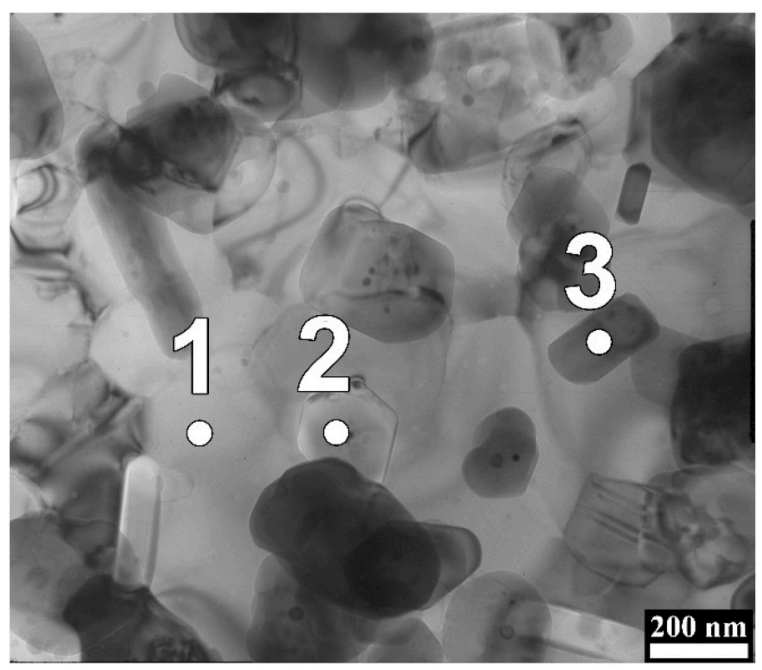

Fig. 5. TEM bright-field image of the AlTiFeCr alloy after SPS and annealing at $1000^{\circ} \mathrm{C}$ for $24 \mathrm{~h}$; typical structural constituents used for chemical analysis (Table 4) are numbered.
Table 4

Chemical composition of the typical structural constituents in AlCrFeTi alloy after SPS and annealing at $1000^{\circ} \mathrm{C}$ for $24 \mathrm{~h}$ (denoted with numbers in Fig. 5), measured by TEM-EDS.

\begin{tabular}{|c|c|c|c|c|c|}
\hline \multicolumn{2}{|c|}{ Structural constituent } & \multicolumn{4}{|c|}{ Elements, at.\% } \\
\hline \# & Designation & $\mathrm{Al}$ & $\mathrm{Ti}$ & $\mathrm{Fe}$ & $\mathrm{Cr}$ \\
\hline 1 & Matrix (C14 Laves) & 19.6 & 29.8 & 31.3 & 19.3 \\
\hline 2 & Light particles (bcc) & 16.0 & 9.4 & 17.4 & 57.2 \\
\hline 3 & Dark particles $\left(\mathrm{L}_{2}\right)$ & 64.5 & 14.9 & 12.3 & 8.3 \\
\hline
\end{tabular}

\section{Discussion}

In the present work, the microstructure and mechanical properties evolution of the AlCrFeTi LDMEA alloy after MA, SPS, and subsequent annealing were examined. Mechanical alloying has resulted in the formation of the amorphous and bcc solid solution phases (Fig. 1). Amortization of the alloy after MA deserves additional attention since it has not been frequently reported for HEAs [40]. Different phenomenological criteria can be used to predict the formation of the different types of phases, including solid solution, intermetallic, and amorphous [41]. For example, there are thermodynamic parameters like the entropy of mixing [5],

$\Delta S_{m i x}=-R \sum c_{i} \ln c_{i}$

where $\mathrm{R}$ is the gas constant and $c_{i}$ is the atomic fraction of element $i$. There is also enthalpy of mixing [42],

$\Delta H_{m i x}=\sum 4 \omega_{i j} c_{i} c_{j}$

where $\omega_{i j}$ is a concentration-dependent interaction parameter between elements $i$ and $j$ in a sub-regular solid solution model [43]. The two parameters above can be combined by thermodynamic parameter $\Omega$ [44],

$\Omega=\mathrm{T}_{\mathrm{m}} \Delta \mathrm{S}_{\mathrm{mix}} /\left|\Delta \mathrm{H}_{\mathrm{mix}}\right|$

where $T_{m}=\sum c_{i} T_{m i}$ and $T_{m i}$ is the melting temperature of element.

Besides, the atomic size difference parameter [42] can also be used,

$\delta r=100 \% \sqrt{\sum c_{i}\left(1-r_{i} / \bar{r}\right)^{2}}$

where $c_{i}$ and $r_{i}$ are the atomic fraction and the atomic radius, respectively, of element $i, \bar{r}=\sum c_{i} r_{i}$ is the average atomic radius. Only solid solution phases were found to form in many HEAs if $\Omega \geq 1.1$ and $\delta r \leq$ $6.6 \%$ [44]. The amorphous structure is expected at lower $\Omega$ and higher $\delta r$. The program AlCrFeTi alloy has $\Omega=1.26$ and $\delta r=6.72$, i.e. almost meets the solid solution structure formation criteria. However, the alloy has been produced using a highly non-equilibrium method, i.e. MA. The formation of amorphous phases in simpler alloys after MA [45,46] (and other non-equilibrium processes [47]) has been frequently reported and was usually attributed to the introduction of different lattice defects during the milling process $[45,46]$. Moreover, the probability of amorphization is a function of not only alloy composition (reflected by equations (3) and (4)), but also of the process parameters (milling intensity, ball-to-powder ratio, etc) [48]. However, the examination of the effect of MA parameters on the structure formation in the program HEA is beyond the scope of the current paper.

More complex microstructure, composed of a mixture of the (Fe, Ti)rich C14 Laves phase, Cr-rich bcc solid solution, and Al-rich $\mathrm{L}_{2}$ phase was found after SPS and subsequent long-term annealing at $1000{ }^{\circ} \mathrm{C}$ (Figs. 1a, 2-5). The amorphous phase after MA is metastable in most alloys and is expected to transform into crystalline, possibly even intermetallic phase(s) after heating at temperatures above a certain level, often called "glass transition" temperature $\left(T_{g}\right)[49,50]$. Generally, $T_{g}$ is about $0.50-0.65 T_{m}\left(T_{m}\right.$ - melting temperature, $T_{m}$ of the AlCrFeTi 


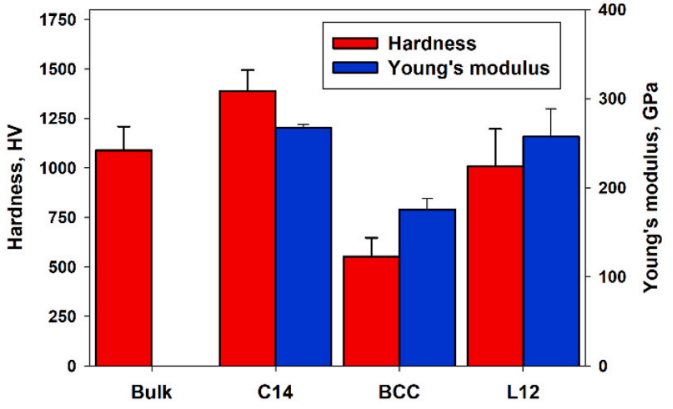

a

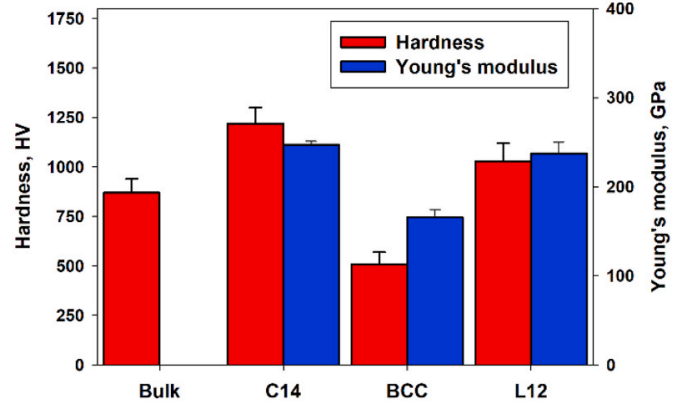

b

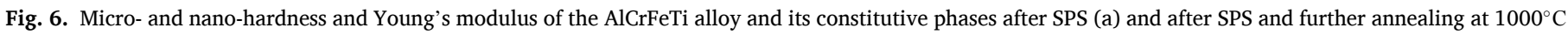
for $24 \mathrm{~h}$ (b).

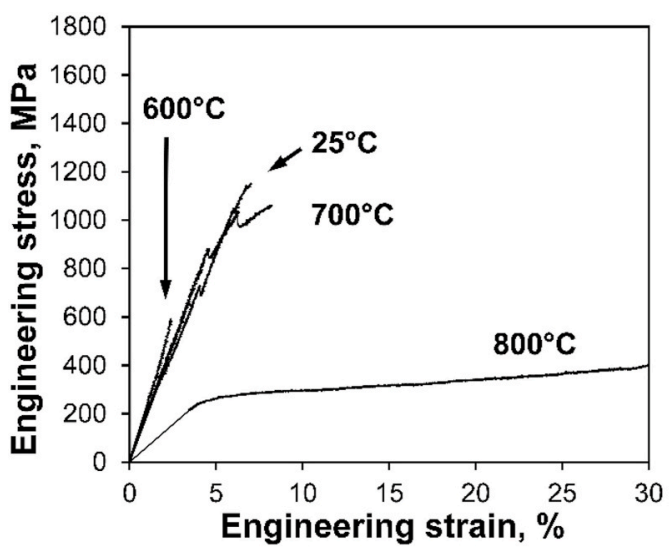

a

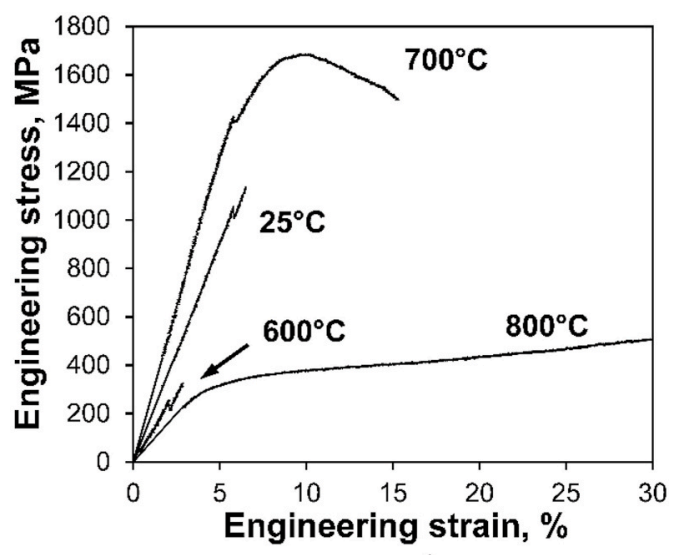

b

Fig. 7. Engineering compression stress-strain curves of the AlCrFeTi alloy after SPS (a) and after SPS and further annealing at $1000^{\circ} \mathrm{C}$ for 24 h (b).

alloy $=1910 \mathrm{~K}$ as per the ThermoCalc results (see below)) [51]. The $T_{g}$ of the AlCrFeTi alloy is obviously lower than SPS temperature $\left(900{ }^{\circ} \mathrm{C}\right.$, $0.61 T_{m}$ ). Therefore, during SPS amorphous phase crystallizes with the formation of several complex phases.

The complex multiphase structure in the AlCrFeTi alloy cannot be readily anticipated from the phenomenological parameters (i.e. $\Omega$ and $\delta r$ ) analysis. Examination of the corresponding binary and ternary phase diagrams have revealed that all the elements had limited mutual solubility; each binary and ternary phase diagram contained one or more intermetallic phase(s) [52]. For instance, the Al-(Cr, Fe, Ti) binaries each contained multiple intermetallic phases, $\mathrm{Cr}-(\mathrm{Fe}, \mathrm{Ti})$ each contained an intermetallic phase ( $\mathrm{FeCr} \sigma$-phase or $\mathrm{Cr}_{2} \mathrm{Ti}$ phase; the latter can have different polymorphs), and the Fe-Ti binary contained two intermetallic phases $\left(\mathrm{Fe}_{2} \mathrm{Ti}\right.$ and $\left.\mathrm{FeTi}\right)$. A powerful tool for analysis of phase stability in such multicomponent alloys is the so-called CALPHAD (CALculation of PHAse Diagrams) [16]. In this work, we have constructed an equilibrium phase diagram $[53,54]$ of the AlCrFeTi alloy using the Thermo-Calc software (Fig. 8, Table 5).

According to the obtained equilibrium phase diagram, the solidification of the alloy starts at $1617^{\circ} \mathrm{C}$ with the bcc phase. The alloy has quite a prolonged solidification interval, and the liquid phase disappears

Table 5

Fractions and chemical compositions of the equilibrium constitutive phases of the AlCrFeTi alloy at $900^{\circ} \mathrm{C}$.

\begin{tabular}{llllll}
\hline \multirow{2}{*}{ Phase } & Fraction & \multicolumn{5}{l}{ Amount of component, at.\% } \\
\cline { 3 - 6 } & & $\mathrm{Al}$ & $\mathrm{Cr}$ & $\mathrm{Fe}$ & $\mathrm{Ti}$ \\
\hline bcc & 0.365 & 25.44 & 47.35 & 12.71 & 14.50 \\
C14 Laves & 0.635 & 23.17 & 12.16 & 32.06 & 32.61 \\
\hline
\end{tabular}

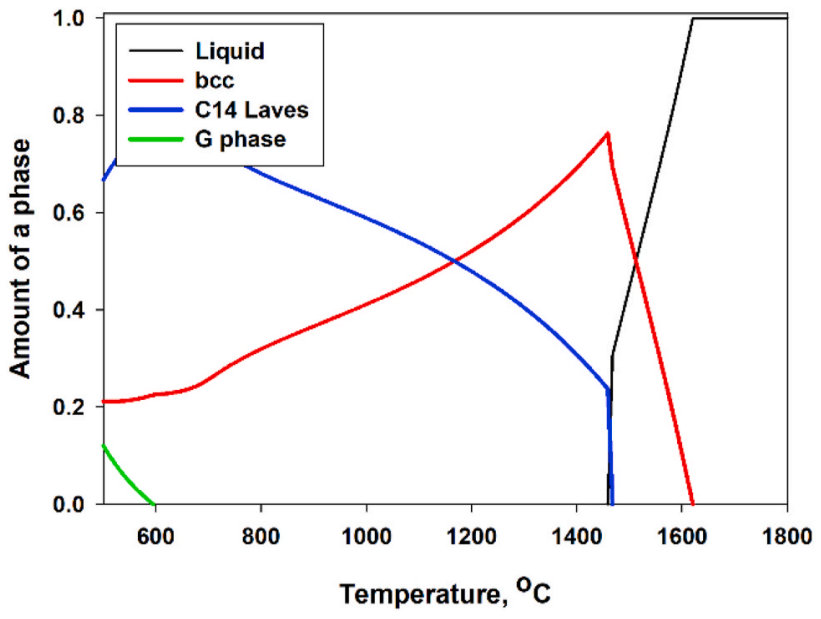

Fig. 8. Equilibrium phase diagram of the AlCrFeTi alloy.

at $1467^{\circ} \mathrm{C}$ via the eutectic $\mathrm{L} \rightarrow \mathrm{bcc}+\mathrm{C} 14$ Laves phase reaction. As a result, the as-solidified alloy must be composed of the primary bcc and the eutectic bcc $+\mathrm{C} 14$ Laves phase mixture. The as-solidified bcc phase is enriched with $\mathrm{Cr}(\sim 29$ at.\%), while the Laves phase is mostly composed of $\mathrm{Fe}$ and $\mathrm{Ti}(\sim 31-34$ at.\%). With a further decrease in temperature, the fraction of the bcc phase decreases and the fraction of the $\mathrm{C} 14$ Laves phase increases. For example, at $900^{\circ} \mathrm{C}$, the ratio between the bcc and Laves phases was $\sim 1: 2$. Cr partitions mainly to the bcc phase: at $900^{\circ} \mathrm{C}$ its concentration in the bcc phase is 47 at. $\%$, while the Laves 
phase contains only 12 at.\% of $\mathrm{Cr}$ (Table 5). The bcc phase is also depleted of $\mathrm{Fe}$ and $\mathrm{Ti}(\sim 13-15$ at.\%), whereas $\mathrm{Al}$ is almost equally distributed between the bcc and Laves phases. A G phase (space group: 225 ) with the chemical composition close to $\mathrm{Al}_{2} \mathrm{FeTi}$ is expected to precipitate at $593{ }^{\circ} \mathrm{C}$.

The experimental phase composition of the AlCrFeTi alloy after SPS and annealing agrees reasonably with the prediction (Fig. 8). For example, the phase diagram correctly predicts C14 Laves as the major phase and the bcc phase as the secondary one; the elemental partitioning is also quite correctly predicted (compare Tables 2, 4 and 5). However, the experimental fraction of the bcc phase $(10-13 \%)$ is lower than the predicted one $(\sim 30 \%)$; besides no Al-rich $\mathrm{L}_{2}$ phase is predicted. Instead, the Al-rich G-phase with much lower solvus temperature $(\sim 600$ ${ }^{\circ} \mathrm{C}$ ) and somewhat different chemical composition is expected to form. These inaccuracies can be attributed to imperfections of the available databases [55]. Although the database used (TCHEA 3.0) contains a full description of all six binaries of the Al-Cr-Fe-Ti system, only one of the ternaries is fully accessed ( $\mathrm{Al}-\mathrm{Fe}-\mathrm{Ti}$ ), two are accessed only partially (Al-Cr-Fe and Al-Cr-Ti), and one is not accessed at all (Cr-Fe-Ti) [56]. Nevertheless, a reasonable agreement between the calculated and experimental results suggests that the CALPHAD approach can be used to predict phases and to optimize the chemical composition of the AlCrFeTi HEA and similar alloys even when they are prepared by MA and SPS.

One of the relatively unexpected findings of the present work is the high stability of the microstructure obtained by SPS during annealing. Although the phase composition of the AlCrFeTi is supposed to be quite stable between 900 and $1000{ }^{\circ} \mathrm{C}$ per the phase diagram (Fig. 8), the structure after SPS contains a high density of grain/interphase boundaries (Fig. 3) that should provide sufficient driving force for structure coarsening [57]. Moreover, the annealing temperature of $1000^{\circ} \mathrm{C}$ corresponds to a rather high homologous temperature of $0.68 T_{m}$. Yet, the average size of grains/particles increased only from 115 to $140 \mathrm{~nm}$ after $24 \mathrm{~h}$ annealing. The relative stability of the microstructure can be attributed to two factors: (i) the ordered C14 Laves matrix phase; (ii) the presence of fine particles of the secondary bcc/L2 1 phases. Coarsening of such a multiphase microstructure requires a diffusive mass transfer. Yet, it is well established that diffusion in intermetallics like the Laves phase is rather slow since random vacancy migration mechanism cannot operate in the ordered materials [63,64]. Besides, the presence of the bcc/L2 1 particles at grain boundaries in the Laves phase matrix can impede their motion due to the well-known Zener drag mechanism [65-68].

Mechanical testing has revealed a high hardness and brittleness of the AlCrFeTi alloy at room temperature (Figs. 6 and 7). This is not surprising given the ordered nature of the matrix Laves phase and the fine-grained microstructure. Some decrease in the hardness of the alloy from 1090 to $870 \mathrm{HV}$ after annealing can be attributed to lower grain boundary strengthening due to the coarsening of the grain/particle size. Also, annealing results in an increment in ductility: after SPS, the alloy exhibited brittle fracture at $700{ }^{\circ} \mathrm{C}$, while in the annealed condition, the alloy becomes ductile at $700^{\circ} \mathrm{C}$. The transition from a brittle to ductile behavior of different HEAs due to heat treatment [58] or thermomechanical processing [59] were reported earlier. In the present case, the increment in ductility after annealing can be attributed to the (i) grain/particle coarsening; (ii) the higher fraction of the softer bcc phase (13 vs $10 \%$ ).

However, the above reasoning cannot explain the simultaneous increase in the strength of the annealed alloy with an increase in the testing temperature. It is known that some alloys exhibit a positive temperature dependence of yield strength on temperature [60]. The well-known example is the alloys with $\mathrm{L} 1_{2}$ structure [61]. In this case, the positive dependence is attributed to the exhaustion of dislocations by cross-slip from $\{111\}$ glide planes to $\{010\}$ planes on which they are sessile. Presumably, the presence of $\mathrm{L} 1_{2}$ particles and positive temperature dependence of their strength can be the reason for the observed

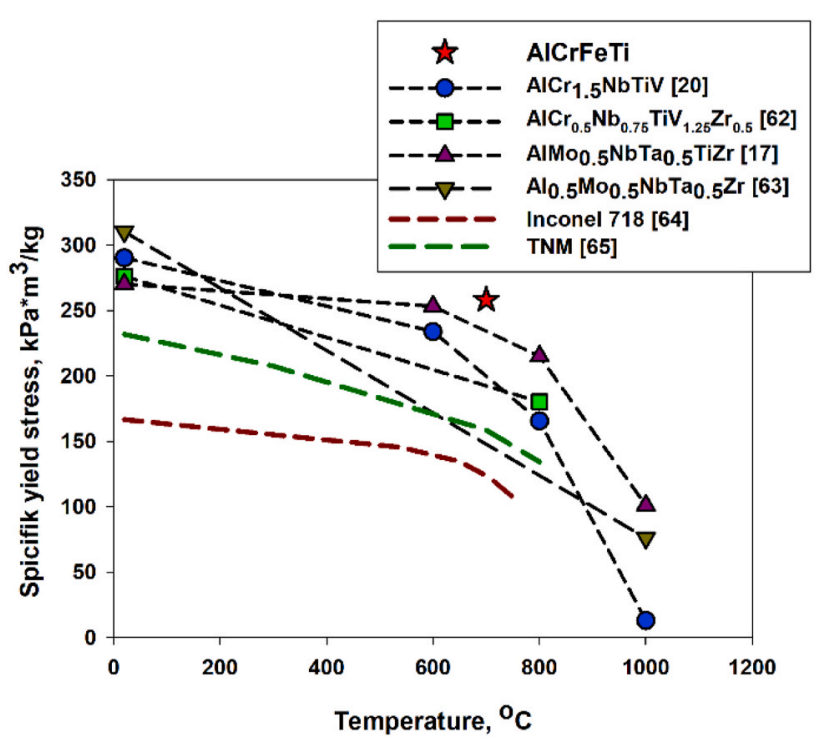

Fig. 9. Comparison between the specific yield strength of the AlCrFeTi alloy with some other HEAs and commercial alloys.

increase of the strength of the AlCrFeTi alloy at $700{ }^{\circ} \mathrm{C}$.

Meanwhile, the annealed $\mathrm{AlCrFeTi}$ alloy demonstrated a very high yield strength of $1430 \mathrm{MPa}$ at $700{ }^{\circ} \mathrm{C}$. Together with a low density of $5.53 \mathrm{~g} \times \mathrm{cm}^{-3}$ this results in an exceptionally high specific strength. The specific yield strength (SYS) at $700{ }^{\circ} \mathrm{C}$ of the AlCrFeTi alloy after annealing is compared in Fig. 9 with the high-temperature properties of the $\mathrm{AlCr}_{1.5} \mathrm{NbTiV}$ [20], $\mathrm{AlCr}_{0.5} \mathrm{Nb}_{0.75} \mathrm{TiV}_{1.25} \mathrm{Zr}_{0.5}$ [62], $\mathrm{AlMo}_{0.5} \mathrm{NbTa}_{0.5}$ TiZr [17], and $\mathrm{Al}_{0.5} \mathrm{Mo}_{0.5} \mathrm{NbTa}_{0.5} \mathrm{Zr}$ [63] RHEAs, as well as with those of commercial Ni-based superalloy Inconel 718 [64] and TiAl-based TNM alloy [65]. It is seen that SYS of the AlCrFeTi alloy at $700{ }^{\circ} \mathrm{C}(258 \mathrm{kPa} \times$ $\mathrm{m}^{3} / \mathrm{kg}$ ) is higher than (extrapolated) SYS of the most capable RHEAs and is superior to commercial materials. This makes AlCrFeTi alloy promising for high-temperature applications. Also note that the alloy is mostly composed of inexpensive elements like $\mathrm{Al}, \mathrm{Cr}$, and $\mathrm{Fe}$, and is likely to have reasonable oxidation resistance due to the high fractions of $\mathrm{Al}$ and $\mathrm{Cr}$. However, further studies are required to examine properties of the alloy in detail and possibly to optimize the alloy composition and processing route to enhance properties (for example, room-temperature ductility).

\section{Conclusions}

In the present work, the microstructure and mechanical properties of the AlCrFeTi medium entropy alloy produced by mechanical alloying and subsequent spark plasma sintering were examined. The following conclusions were drawn:

Mechanical alloying produced the AlCrFeTi powder with a homogeneous chemical composition composed of amorphous and bcc phases. Consolidation of the obtained powder by spark plasma sintering at 900 ${ }^{\circ} \mathrm{C}$ resulted in the production of dense bulk specimens. The density of the alloy was $5.53 \pm 0.07 \mathrm{~g} \times \mathrm{cm}^{-3}$. Sintering has also resulted in the formation of the (Fe, Ti)-rich C14 Laves matrix phase and secondary $\mathrm{Cr}$-rich bcc phase and Al-rich $\mathrm{L}_{2}$ phase. The phase composition of the sintered alloy was in reasonable agreement with the ThermoCalc predictions. Annealing at $1000^{\circ} \mathrm{C}$ has resulted in both coarsening of grains/particles and an increase in the fraction of the secondary phases; however, quite minor changes suggest rather high stability of the microstructure.

The sintered and annealed AlCrFeTi alloy had a high hardness of 870-1090 HV and pronounced brittleness at room temperature. The alloy after SPS demonstrated compression ductility at only $800^{\circ} \mathrm{C}$, while after annealing the alloy became ductile at $700{ }^{\circ} \mathrm{C}$. Moreover, the annealed alloy demonstrated high yield strength of $1430 \mathrm{MPa}$ and 
exceptional specific yield strength of $258 \mathrm{kPa} \times \mathrm{m}^{3} / \mathrm{kg}$, superior to other high entropy alloys and commercial high-temperature alloys. However, the alloy softened considerably at $800^{\circ} \mathrm{C}$.

\section{Data availability}

The raw/processed data required to reproduce these findings cannot be shared at this time as the data also forms part of an ongoing study.

\section{CRediT authorship contribution statement}

D. Shaysultanov: Investigation. A. Nepapushev: Investigation, Writing - review \& editing. S. Zherebtsov: Methodology, Writing - review \& editing. D. Moskovskikh: Supervision, Writing - review \& editing, Funding acquisition. N. Stepanov: Writing - review \& editing, Writing - original draft, Investigation, Writing - original draft, Visualization, Supervision, Funding acquisition.

\section{Declaration of competing interest}

The authors declare that they have no known competing financial interests or personal relationships that could have appeared to influence the work reported in this paper.

\section{Acknowledgments}

The authors gratefully acknowledge the financial support from the Russian Foundation for Basic Research (Grant № 18-38-20013) and the Ministry of Science and Higher Education of the Russian Federation in the framework of Increase Competitiveness Program of NUST «MISiS» (№ K2-2019-007), implemented by a governmental decree dated March $16,2013, \mathrm{~N} 211$. The authors are grateful to the personnel of the Joint Research Center, «Technology and Materials», Belgorod State National Research University, for their assistance with the instrumental analysis.

\section{References}

[1] E.P. George, D. Raabe, R.O. Ritchie, High-entropy alloys, Nat. Rev. Mater. (2019) 1, https://doi.org/10.1038/s41578-019-0121-4.

[2] Y. Zhang, T.T. Zuo, Z. Tang, M.C. Gao, K.A. Dahmen, P.K. Liaw, Z.P. Lu, Microstructures and properties of high-entropy alloys, Prog. Mater. Sci. 61 (2014), https://doi.org/10.1016/j.pmatsci.2013.10.001.

[3] E.J. Pickering, N.G. Jones, High-entropy alloys: a critical assessment of their founding principles and future prospects, Int. Mater. Rev. (2016) 183-202, https:// doi.org/10.1080/09506608.2016.1180020.

[4] D.B.B. Miracle, O.N.N. Senkov, A critical review of high entropy alloys and related concepts, Acta Mater. 122 (2017) 448-511, https://doi.org/10.1016/j. actamat.2016.08.081.

[5] J.W.J.-W. Yeh, S.-K.S.K. Chen, S.J.S.-J. Lin, J.Y.J.-Y. Gan, T.S.T.-S. Chin, T.-T.T. T. Shun, C.H.C.-H. Tsau, S.Y.S.-Y. Chang, Nanostructured high-entropy alloys with multiple principal elements: novel alloy design concepts and outcomes, Adv. Eng. Mater. 6 (2004) 299-303+274, https://doi.org/10.1002/adem.200300567.

[6] S. Gorsse, D.B. Miracle, O.N. Senkov, Mapping the world of complex concentrated alloys, Acta Mater. 135 (2017) 177-187, https://doi.org/10.1016/j. actamat.2017.06.027.

[7] Z. Li, S. Zhao, R.O. Ritchie, M.A. Meyers, Mechanical properties of high-entropy alloys with emphasis on face-centered cubic alloys, Prog. Mater. Sci. 102 (2019) 296-345, https://doi.org/10.1016/j.pmatsci.2018.12.003.

[8] F. Otto, A. Dlouhý, C. Somsen, H. Bei, G. Eggeler, E.P. George, The influences of temperature and microstructure on the tensile properties of a CoCrFeMnNi highentropy alloy, Acta Mater. 61 (2013) 5743-5755, https://doi.org/10.1016/j. actamat.2013.06.018.

[9] B. Cantor, I.T.H. Chang, P. Knight, A.J.B. Vincent, Microstructural development in equiatomic multicomponent alloys, Mater. Sci. Eng. 375 (2004) 213-218, https:// doi.org/10.1016/j.msea.2003.10.257.

[10] B. Gludovatz, A. Hohenwarter, D. Catoor, E.H. Chang, E.P. George, R.O. Ritchie, A fracture-resistant high-entropy alloy for cryogenic applications, Science 345 (80) (2014) 1153-1158, https://doi.org/10.1126/science.1254581.

[11] B. Gwalani, V. Soni, M. Lee, S. Mantri, Y. Ren, R. Banerjee, Optimizing the coupled effects of Hall-Petch and precipitation strengthening in a Al0.3CoCrFeNi high entropy alloy, Mater. Des. 121 (2017) 254-260, https://doi.org/10.1016/j. matdes.2017.02.072.

[12] N.D. Stepanov, D.G. Shaysultanov, R.S. Chernichenko, D.M. Ikornikov, V.N. Sanin, S.V. Zherebtsov, Mechanical properties of a new high entropy alloy with a duplex ultra-fine grained structure, Mater. Sci. Eng. 728 (2018) 54-62, https://doi.org/ 10.1016/j.msea.2018.04.118.

[13] M.V. Klimova, D.G. Shaysultanov, S.V. Zherebtsov, N.D. Stepanov, Effect of second phase particles on mechanical properties and grain growth in a CoCrFeMnNi high entropy alloy, Mater. Sci. Eng. 748 (2019) 228-235, https://doi.org/10.1016/J. MSEA.2019.01.112.

[14] J.Y. He, H. Wang, H.L. Huang, X.D. Xu, M.W. Chen, Y. Wu, X.J. Liu, T.G. Nieh, K. An, Z.P. Lu, A precipitation-hardened high-entropy alloy with outstanding tensile properties, Acta Mater. 102 (2016) 187-196, https://doi.org/10.1016/j. actamat.2015.08.076.

[15] O.N. Senkov, D.B. Miracle, K.J. Chaput, J.-P. Couzinie, Development and exploration of refractory high entropy alloys-a review, J. Mater. Res. 33 (2018) 3092-3128, https://doi.org/10.1557/jmr.2018.153.

[16] D.B. Miracle, J.D. Miller, O.N. Senkov, C. Woodward, M.D. Uchic, J. Tiley, Exploration and development of high entropy alloys for structural applications, Entropy 16 (2014) 494-525, https://doi.org/10.3390/e16010494.

[17] O.N. Senkov, D. Isheim, D.N. Seidman, A.L. Pilchak, Development of a refractory high entropy superalloy, Entropy 18 (2016), https://doi.org/10.3390/e18030102.

[18] O.N. Senkov, S.V. Senkova, D.B. Miracle, C. Woodward, Mechanical properties of low-density, refractory multi-principal element alloys of the $\mathrm{Cr}-\mathrm{Nb}-\mathrm{Ti}-\mathrm{V}-\mathrm{Zr}$ system, Mater. Sci. Eng. 565 (2013) 51-62, https://doi.org/10.1016/j. msea.2012.12.018.

[19] N.Y. Yurchenko, N.D. Stepanov, D.G. Shaysultanov, M.A. Tikhonovsky, G. A. Salishchev, Effect of Al content on structure and mechanical properties of the AlxCrNbTiVZr ( $x=0 ; 0.25 ; 0.5 ; 1)$ high-entropy alloys, Mater. Char. 121 (2016) 125-134, https://doi.org/10.1016/j.matchar.2016.09.039.

[20] N.D. Stepanov, N.Y. Yurchenko, D.V. Skibin, M.A. Tikhonovsky, G.A. Salishchev, Structure and mechanical properties of the AlCrxNbTiV $(x=0,0.5,1,1.5)$ high entropy alloys, J. Alloys Compd. 652 (2015) 266-280, https://doi.org/10.1016/j. jallcom.2015.08.224.

[21] K.M. Youssef, A.J. Zaddach, C. Niu, D.L. Irving, C.C. Koch, A novel low-density, high-hardness, high-entropy alloy with close-packed single-phase nanocrystalline structures, Mater. Res. Lett. 3 (2015) 95-99, https://doi.org/10.1080/ 21663831.2014.985855.

[22] O. Maulik, D. Kumar, S. Kumar, S.K. Dewangan, V. Kumar, Structure and properties of lightweight high entropy alloys: a brief review, Mater. Res. Express 5 (2018), https://doi.org/10.1088/2053-1591/aabbca.

[23] A. Kumar, M. Gupta, An insight into evolution of light weight high entropy alloys: a review, Metals 6 (2016), https://doi.org/10.3390/met6090199.

[24] R. Feng, M.C. Gao, C. Zhang, W. Guo, J.D. Poplawsky, F. Zhang, J.A. Hawk, J. C. Neuefeind, Y. Ren, P.K. Liaw, Phase stability and transformation in a lightweight high-entropy alloy, Acta Mater. (2018), https://doi.org/10.1016/j. actamat.2017.12.061.

[25] Y.C. Liao, T.H. Li, P.H. Tsai, J.S.C. Jang, K.C. Hsieh, C.Y. Chen, J.C. Huang, H. J. Wu, Y.C. Lo, C.W. Huang, I.Y. Tsao, Designing novel lightweight, high-strength and high-plasticity Ti (AlCrNb)100- medium-entropy alloys, Intermetallics 117 (2020) 106673, https://doi.org/10.1016/j.intermet.2019.106673.

[26] N.D. Stepanov, D.G. Shaysultanov, G.A. Salishchev, M.A. Tikhonovsky, Structure and mechanical properties of a light-weight AlNbTiV high entropy alloy, Mater. Lett. 142 (2015) 153-155, https://doi.org/10.1016/j.matlet.2014.11.162.

[27] W. Sun, X. Huang, A.A. Luo, Phase formations in low density high entropy alloys, Calphad Comput. Coupling Phase Diagrams Thermochem. 56 (2017) 19-28, https://doi.org/10.1016/j.calphad.2016.11.002.

[28] K. Tseng, Y. Yang, C. Juan, T. Chin, C. Tsai, J. Yeh, A light-weight high-entropy alloy Al20Be20Fe10Si15Ti35, Sci. China Technol. Sci. 61 (2018) 184-188, https:// doi.org/10.1007/s11431-017-9073-0.

[29] X.H. Du, R. Wang, C. Chen, B.L. Wu, J.C. Huang, Preparation of a light-weight MgCaAlLiCu high-entropy alloy, Key Eng. Mater. 727 (2017) 132-135, https://doi, org/10.4028/www.scientific.net/KEM.727.132.

[30] Z. Fu, W. Chen, H. Wen, D. Zhang, Z. Chen, B. Zheng, Y. Zhou, E.J. Lavernia, Microstructure and strengthening mechanisms in an FCC structured single-phase nanocrystalline Co25Ni25Fe25Al7.5Cu17.5 high-entropy alloy, Acta Mater. 107 (2016) 59-71, https://doi.org/10.1016/j.actamat.2016.01.050.

[31] Z. Fu, A. Hoffman, B.E. MacDonald, Z. Jiang, W. Chen, M. Arivu, H. Wen, E. J. Lavernia, Atom probe tomography study of an Fe25Ni25Co25Ti15Al10 highentropy alloy fabricated by powder metallurgy, Acta Mater. 179 (2019) 372-382, https://doi.org/10.1016/J.ACTAMAT.2019.08.047.

[32] B. Kang, T. Kong, A. Raza, H.J. Ryu, S.H. Hong, Fabrication, microstructure and mechanical property of a novel Nb-rich refractory high-entropy alloy strengthened by in-situ formation of dispersoids, Int. J. Refract. Metals Hard Mater. (2019), https://doi.org/10.1016/J.IJRMHM.2019.02.009.

[33] B. Kang, J. Lee, H.J. Ryu, S.H. Hong, Ultra-high strength WNbMoTaV high-entropy alloys with fine grain structure fabricated by powder metallurgical process, 712 , 2018, pp. 616-624. https://www.sciencedirect.com/science/article/pii/S0921509 317316192. (Accessed 13 February 2018).

[34] I. Moravcik, L. Gouvea, V. Hornik, Z. Kovacova, M. Kitzmantel, E. Neubauer, I. Dlouhy, Synergic strengthening by oxide and coherent precipitate dispersions in high-entropy alloy prepared by powder metallurgy, Scripta Mater. 157 (2018) 24-29, https://doi.org/10.1016/J.SCRIPTAMAT.2018.07.034.

[35] A.S. Rogachev, S.G. Vadchenko, N.A. Kochetov, S. Rouvimov, D.Y. Kovalev, A. S. Shchukin, D.O. Moskovskikh, A.A. Nepapushev, A.S. Mukasyan, Structure and properties of equiatomic CoCrFeNiMn alloy fabricated by high-energy ball milling and spark plasma sintering, J. Alloys Compd. 805 (2019) 1237-1245, https://doi. org/10.1016/j.jallcom.2019.07.195. 
[36] S. Yadav, K. Biswas, A. Kumar, Spark plasma sintering of high entropy alloys, in: Spark Plasma Sinter. Mater, Springer International Publishing, 2019, pp. 539-571, https://doi.org/10.1007/978-3-030-05327-7_19.

[37] S. Varalakshmi, M. Kamaraj, B.S. Murty, Synthesis and characterization of nanocrystalline AlFeTiCrZnCu high entropy solid solution by mechanical alloying, J. Alloys Compd. 460 (2008) 253-257, https://doi.org/10.1016/J. JALLCOM.2007.05.104.

[38] A.S. Rogachev, D.O. Moskovskikh, A.A. Nepapushev, T.A. Sviridova, S. G. Vadchenko, S.A. Rogachev, A.S. Mukasyan, Experimental investigation of milling regimes in planetary ball mill and their influence on structure and reactivity of gasless powder exothermic mixtures, Powder Technol. 274 (2015) 44-52, https://doi.org/10.1016/j.powtec.2015.01.009.

[39] W.C. Oliver, G.M. Pharr, Measurement of hardness and elastic modulus by instrumented indentation: advances in understanding and refinements to methodology, J. Mater. Res. 19 (2004) 3-20, https://doi.org/10.1557/ jmr.2004.19.1.3.

[40] Y.L. Chen, C.W. Tsai, C.C. Juan, M.H. Chuang, J.W. Yeh, T.S. Chin, S.K. Chen, Amorphization of equimolar alloys with HCP elements during mechanical alloying, J. Alloys Compd. 506 (2010) 210-215, https://doi.org/10.1016/j. jallcom.2010.06.179.

[41] Z. Wang, S. Guo, C.T. Liu, Phase selection in high-entropy alloys: from nonequilibrium to equilibrium, JOM (J. Occup. Med.) 66 (2014), https://doi.org/ 10.1007/s11837-014-0953-8.

[42] Y. Zhang, Y.J. Zhou, J.P. Lin, G.L. Chen, P.K. Liaw, Solid-solution phase formation rules for multi-component alloys, Adv. Eng. Mater. 10 (2008) 534-538, https:// doi.org/10.1002/adem.200700240.

[43] A. Takeuchi, A. Inoue, Classification of bulk metallic glasses by atomic size difference, heat of mixing and period of constituent elements and its application to characterization of the main alloying element, Mater. Trans. 46 (2005) 2817-2829, https://doi.org/10.2320/matertrans.46.2817.

[44] X. Yang, Y. Zhang, Prediction of high-entropy stabilized solid-solution in multicomponent alloys, Mater. Chem. Phys. 132 (2012) 233-238, https://doi.org/ 10.1016/j.matchemphys.2011.11.021.

[45] C.C. Koch, Amorphization by mechanical alloying, J. Non-Cryst. Solids 117-118 (1990) 670-678, https://doi.org/10.1016/0022-3093(90)90620-2.

[46] E. Hellstern, L. Schultz, Amorphization of transition metal $\mathrm{Zr}$ alloys by mechanical alloying, Appl. Phys. Lett. 48 (1986) 124-126, https://doi.org/10.1063/1.96971.

[47] M. Ghidelli, H. Idrissi, S. Gravier, J.J. Blandin, J.P. Raskin, D. Schryvers, T. Pardoen, Homogeneous flow and size dependent mechanical behavior in highly ductile Zr65Ni35 metallic glass films, Acta Mater. 131 (2017) 246-259, https:// doi.org/10.1016/j.actamat.2017.03.072.

[48] B.S. Murty, M. Mohan Rao, S. Ranganathan, Milling maps and amorphization during mechanical alloying, Acta Metall. Mater. 43 (1995) 2443-2450, https://doi. org/10.1016/0956-7151(94)00402-1.

[49] M. Kambara, K. Uenishi, K.F. Kobayashi, Nano-structured intermetallic compound TiAl obtained by crystallization of mechanically alloyed amorphous TiAl, and its subsequent grain growth, J. Mater. Sci. 35 (2000) 2897-2905, https://doi.org/ 10.1023/A:1004771808047.
[50] Y.J. Liu, I.T.H. Chang, The correlation of microstructural development and thermal stability of mechanically alloyed multicomponent Fe-Co-Ni-Zr-B alloys, Acta Mater. 50 (2002) 2747-2760, https://doi.org/10.1016/S1359-6454(02)00118-0.

[51] A.L. Greer, Crystallization of amorphous alloys, Metall. Mater. Trans. A Phys. Metall. Mater. Sci. 27 (1996) 549-555, https://doi.org/10.1007/BF02648945.

[52] SpringerMaterials - properties of materials, n.d. https://materials.springer.com/. (Accessed 6 November 2019).

[53] E. Nes, N. Ryum, O. Hunderi, On the Zener drag, Acta Metall. 33 (1985) 11-22, https://doi.org/10.1016/0001-6160(85)90214-7.

[54] M. Hillert, Inhibition of grain growth by second-phase particles, Acta Metall. 36 (1988) 3177-3181, https://doi.org/10.1016/0001-6160(88)90053-3.

[55] K.N. Wertz, J.D. Miller, O.N. Senkov, Toward multi-principal component alloy discovery: assessment of CALPHAD thermodynamic databases for prediction of novel ternary alloy systems, J. Mater. Res. (2018) 1-14, https://doi.org/10.1557/ jmr.2018.61.

[56] No title, n.d. https://www.thermocalc.com/media/54070/tchea3_extended_info. pdf. (Accessed 6 November 2019).

[57] T. Chookajorn, H.A. Murdoch, C.A. Schuh, Design of stable nanocrystalline alloys, Science 337 (80) (2012) 951-954, https://doi.org/10.1126/science.1224737.

[58] V. Soni, O.N. Senkov, B. Gwalani, D.B. Miracle, R. Banerjee, Microstructural design for improving ductility of an initially brittle refractory high entropy alloy, Sci. Rep. 8 (2018) 8816, https://doi.org/10.1038/s41598-018-27144-3.

[59] A.V. Kuznetsov, D.G. Shaysultanov, N.D. Stepanov, G.A. Salishchev, O.N. Senkov, Tensile properties of an AlCrCuNiFeCo high-entropy alloy in as-cast and wrought conditions, Mater. Sci. Eng. 533 (2012) 107-118, https://doi.org/10.1016/j. msea.2011.11.045.

[60] S.M. Hosseini, P. Novák, M. Veselý, Positive temperature dependence of compressive properties in an AlNiCo poly-quasicrystal fabricated by mechanical alloying and spark plasma sintering, Scripta Mater. 187 (2020) 169-174, https:// doi.org/10.1016/j.scriptamat.2020.06.023.

[61] Y.M. Wang-Koh, Understanding the yield behaviour of L12-ordered alloys, Mater. Sci. Technol. 33 (2017) 934-943, https://doi.org/10.1080/ 02670836.2016 .1215961$.

[62] N. Yurchenko, E. Panina, M. Tikhonovsky, G. Salishchev, S. Zherebtsov, N. Stepanov, Structure and mechanical properties of an in situ refractory Al20Cr10Nb15Ti20V25Zr10 high entropy alloy composite, Mater. Lett. 264 (2020) 127372, https://doi.org/10.1016/j.matlet.2020.127372.

[63] O.N. Senkov, J.K. Jensen, A.L. Pilchak, D.B. Miracle, H.L. Fraser, Compositional variation effects on the microstructure and properties of a refractory high-entropy superalloy AlMo0.5NbTa0.5TiZr, Mater. Des. 139 (2018) 498-511, https://doi. org/10.1016/j.matdes.2017.11.033.

[64] No title, n.d, https://www.specialmetals.com/assets/smc/documents/inconel_allo y_718.pdf. (Accessed 27 March 2020).

[65] E. Schwaighofer, H. Clemens, S. Mayer, J. Lindemann, J. Klose, W. Smarsly, V. Güther, Microstructural design and mechanical properties of a cast and heattreated intermetallic multi-phase $\gamma$-TiAl based alloy, Intermetallics 44 (2014) 128-140, https://doi.org/10.1016/j.intermet.2013.09.010. 